\title{
EVALUATING VARIOUS APPROACHES TO IMPROVE ROAD INFRASTRUCTURE AND CONTROLLING PEDESTRIAN CROSSINGS IN INDIA
}

\author{
M. Durga \\ Civil Engineering Department, \\ Noida International University, Greater Noida, India \\ Dr. Paritosh Srivastava \\ Associate Professor, HOD -Civil Engineering, \\ Noida International University (NIU), Greater Noida, India \\ Dr. A. Shanmuganathan \\ Associate Professor, Department of Mechanical Engineering, \\ School of Engineering \& Technology, Noida International University, India
}

\begin{abstract}
The study projects the various aspects of development of pedestrian crossings such that the people of India would be able to facilitate a safety measure during a crossing of congested roads. The study also provides relevant measures that are to be adopted by the government of India to develop the mentioned proposal. Dealing with the various development mechanisms, it could be stated that applying time scheduled signal posts at every crossing would eventually enhance the traffic handling ability of the governing bodies as well as provide an estimation of the controlling of pedestrian publics while crossing the roads. The study also draws conclusions based on the evaluation of the methods and technologies along with the projection of the futuristic benefits of the application.
\end{abstract}

Keywords: Pedestrian, Safety Measure, Traffic.

Cite this Article: M. Durga, Paritosh Srivastava and A. Shanmuganathan, Evaluating Various Approaches to Improve Road Infrastructure and Controlling Pedestrian Crossings in India. International Journal of Civil Engineering and Technology (IJCIET), 11(1), 2020, 146-157. https://iaeme.com/Home/issue/IJCIET?Volume=11\&Issue $=1$

\section{INTRODUCTION}

Pedestrian crossing plays an important role in the transportation of people as a mid-block chain to connect roads from one direction to another. The optimization of this pedestrian 
crossing eventually benefits the vehicles as well as the individuals to provide time-effective methods of transportation. According to the present condition of Indian traffic, it has been observed that there exists immense congestion in the pedestrian crossings during the morning and evening peak hours. The study puts forward various ways to improve the conditions of the traffic through the improvement of pedestrian crossings such that the average waiting time for the vehicles would be eventually reduced.

\subsection{Background of the Study}

The study has been conducted to address the various issues that the Indian traffic faces due to the ineffective application of pedestrian crossings. It has been advised that the inappropriate placement of the pedestrian crossings speciously in the junction sections of the roads had led the Indian traffic to face congestions. The study aims to provide several reasons for the development of pedestrian crossings to improve the transportation facilities of individuals.

\subsection{Rationale}

The ineffective planning of the pedestrian development of the roads of India has been a problem for decades. With the proper application of strategies, the development of the pedestrian crossings would be evaluated. Through this study, various aspects of development have been visited that satisfied the reasonable benefits of applying optimized pedestrian coursing development to improve traffic conditions of the Indian subcontinent.

\subsection{Aim}

The study aims to provide solutions in the form of optimization techniques to address the issues that India faces due to inappropriate management of the pedestrian crossing and road infrastructure.

\subsection{Objectives}

- To understand the issues faced related to inappropriate road infrastructure and pedestrian crossings

- To initiate the development of optimization techniques to improve road infrastructure and pedestrian crossings

- To recommend future plans in implementing modern technologies to optimize pedestrian crossings and develop road infrastructure

\subsection{Research Questions}

- What are the various issues related to the inefficient road infrastructure and pedestrian crossing systems in India?

- What are the various optimization techniques that could be applied to improve road infant suture and pedestrian crossings?

- What are the factors that lead to congestion in pedestrian crossings?

- How would modern technologies help in mitigating the issues related to road infrastructure improvement?

- How would the optimization techniques benefit individuals in the future? 


\section{LITERATURE REVIEW}

\subsection{Conceptual Framework}

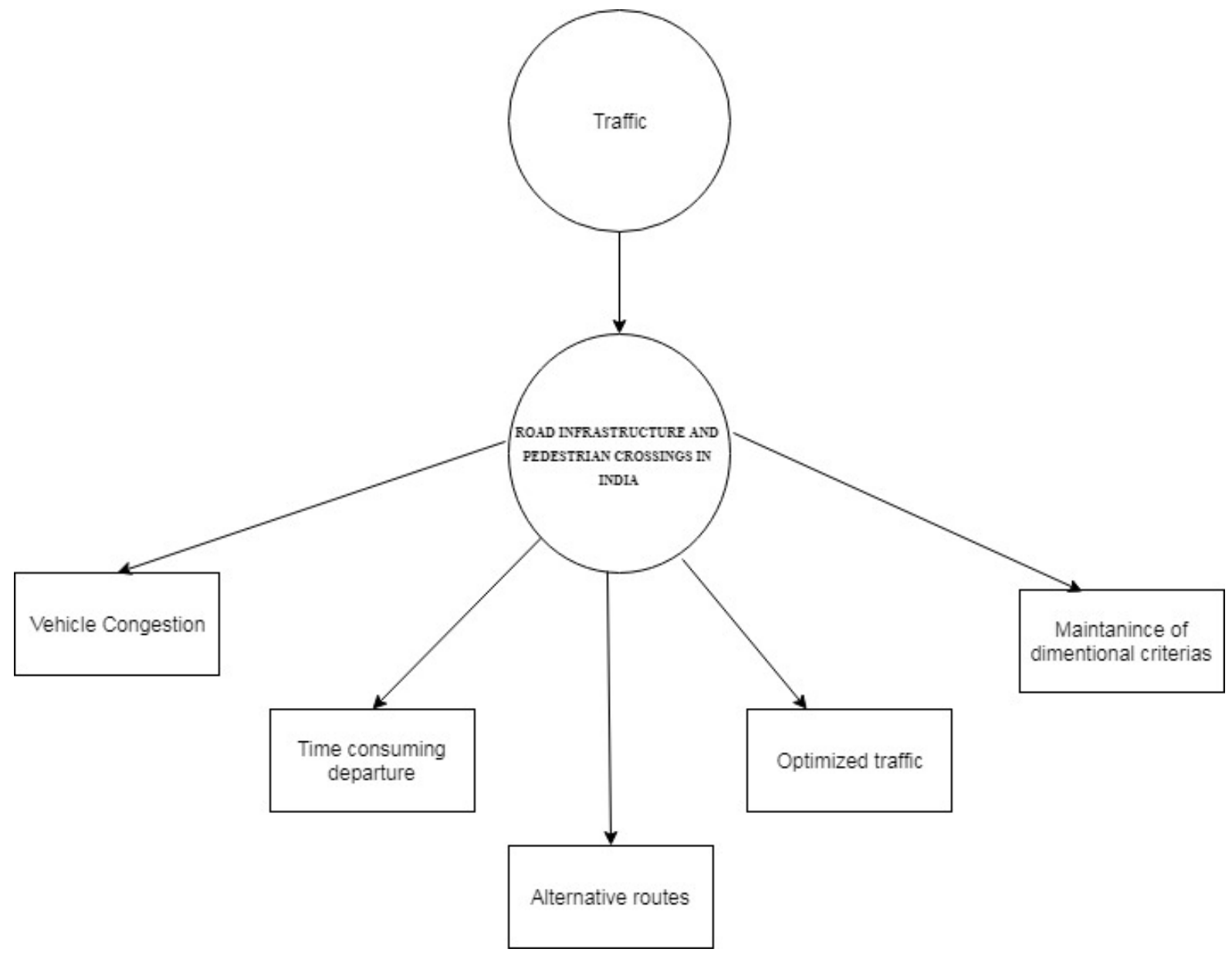

Figure 1 Conceptual Framework

\subsection{Road Traffic and Pedestrian Congestion Issues in India}

According to the various studies that have been conducted, it has been observed that inappropriate and unplanned structured off the road and pedestrian crossings are one of the main reasons that lead to the congestion in traffic in India. The development of efficient road infrastructure has been said to be the one and only improvement method that could be applied to address and mitigate the issue ${ }^{[1]}$. The following factors have been identified that lead to the stated problem.

- Several accidents have been observed during recent years that have been found to be ineffective management of pedestrian crossings.

- Overcrowding situations near the narrow paths.

- Discomfort for both entities including the individuals as well as the traffic maintainers.

- During peak hours there exist huge traffic especially in the junction areas of the cities of India.

- Time-consuming situations release due to congestion in the crossing regions situated near bus terminals.

- Too many vehicles at a particular position cause a huge amount of exhaust released into the atmosphere causing pollution. 
- Aged people within the congested areas may face breathing issues and may suffocate.

Taking into account the stated issues, several methods and techniques have been addressed through these strategies to improve the conditions. In this way, not only the traffic conditions would be improved but also enhances the development of the cities in a wellstructured way.

\subsection{Importance of Pedestrian Crossing}

Pedestrian crossings have been observed to be one of the most important components of road users in India. The issues identified in the previous section directly indicate the importance of having a well-structured pedestrian crossing to benefit the individuals to have an optimized road transport facility ${ }^{[4]}$. In order to identify the various benefits of having effective pedestrian crossings, there needs to be a complete understanding of the types of pedestrian crossing and their significance.

According to the various theoretical aspects, two types of pedestrian crossing technical have been identified based on their usability and applications, one of which is an at-grade pedestrian crossing and the other is a grade-separated pedestrian crossing. Evaluating the atgrade pedestrian crossing provides relevant measures both as a controlled and uncontrolled manner ${ }^{[12]}$. Zebra crossing, signal-controlled crossing, and police maintained crossing are categorized under the controlled measures, whereas, paint lines and studs are considered as the uncontrolled pedestrian crossing mechanisms. Grade separated pedestrian crossing on the other and provides the governing bodies with the measures to apply footbridges and subways to mitigate traffic issues ${ }^{[10]}$. However, the application of this method uses several consecutive methods and thereby increasing the time delay of the traveling individuals.

Apart from having a disadvantageous feature of the grade-separated pedestrian crossing, it has been observed to be safe and secluded from both the vehicular transports as well as the individuals. Taking into account the importance of implementing appropriate pedestrian crossing within the traffic conditions of India, this would enable the governing bodies as well as the vehicles and other transportation manage their time along with efficient management of traffic.

\subsection{Optimization Techniques}

Considering one of the effective mechanisms, the application of time scheduled crossing mechanisms through traffic signals, there would be a diversified nature benefitting both the individual as well as the drivers to facilitate from time issues. The time-based scheduling, in this case, provides the divers with an estimated waiting time along with the border of road crossing ${ }^{[7]}$. Within this time, the individuals are suggested to cross the roads. The availability of a time display helps the individuals as well as the divers to estimate their departure from the post ${ }^{[11]}$. This, in turn, helps in maintaining a lot of vehicles as well as individuals eliminating the unorganized issues. However, this method may seem effective but consists of several flaws. Taking into account of one of the frequently occurring situations, often young people fail to decide their ability to cross the road within the remaining signal toggle time. In these cases, the occurrence of accidents is to the maximum ${ }^{[22]}$. Therefore, the government is suggested to apply for more effective mechanisms where the allocated time for crossing the road is determined distinctly. 
Evaluating Various Approaches to Improve Road Infrastructure and Controlling Pedestrian Crossings in India

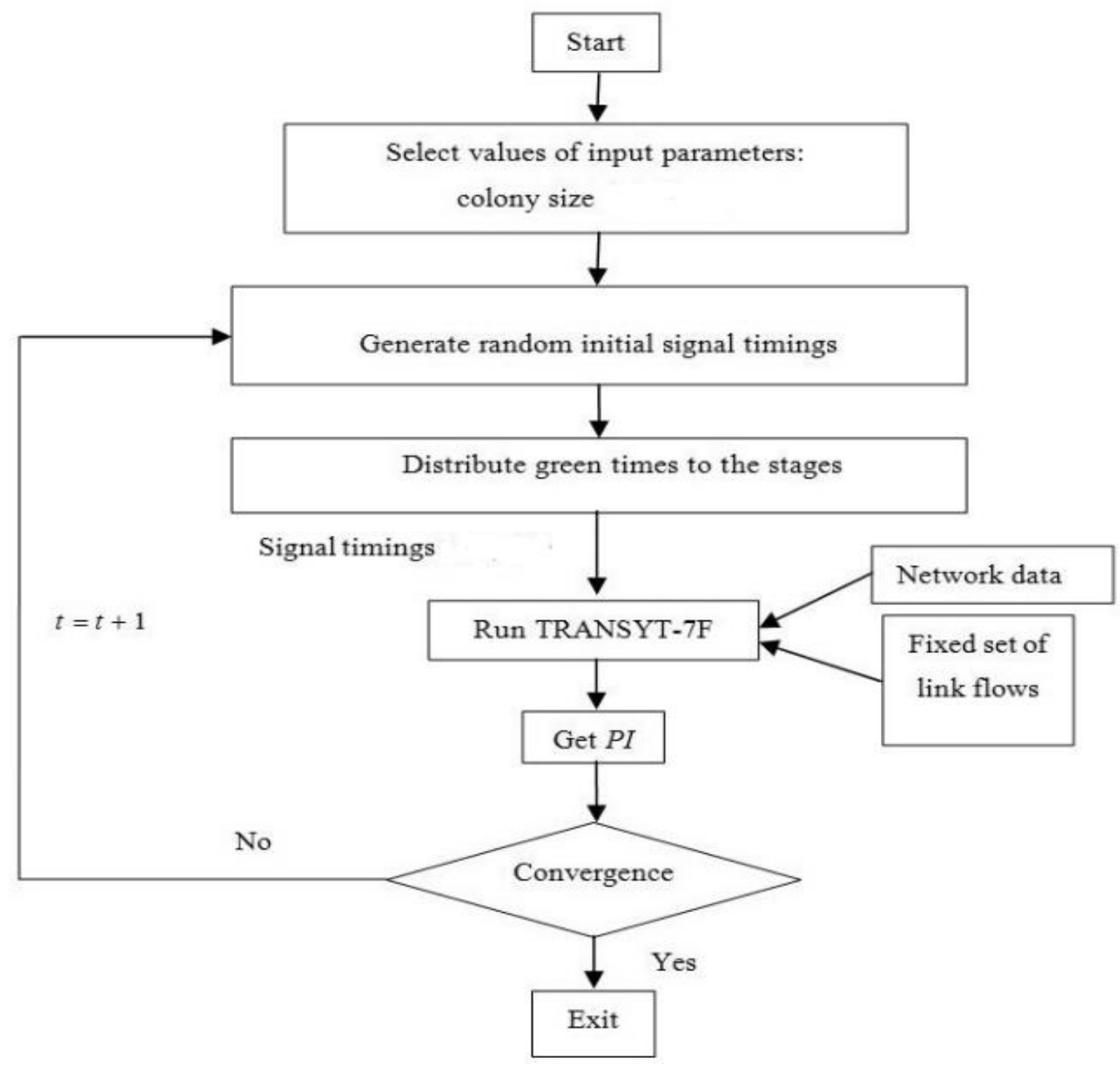

Figure 2 Flowchart of time scheduled crossing mechanism

(Source: Influenced by ${ }^{[7]}$ )

\subsection{Benefits of Optimization}

Surveys from all over sectional analysis of the cities of India, especially in the region of the metropolitan cities have been observed an unpleasant experience of people traveling during the peak hours. In other words, the overcrowding situation near the junctions of the streets often results in risky ways of crossing roads ${ }^{[17]}$. The development of pedestrian crossings would lead not only the traffic governors but also the road users to benefit from traveling. Several techniques are available to the government that addresses solutions to the issues. For instance, many cities in India have been observed to be using subways and footbridges to cross broad streets. This results in a safe way of developing road crossing facilities. On the contrary, people who often prefer shortcuts rather than time-consuming mechanisms are observed to be using on a road crossing behavior ${ }^{[18]}$. Therefore, there needs to be a severe development of efficient techniques that would help the individuals to crossroads effectively and safely. On the other hand, the presence of traffic police as the coordinator of the vehicular transport would help the people in doing the same ${ }^{[17]}$. Concluding from the perspectives of drivers, a mass number of individuals crossing roads directly create congestion to the vehicles 
and therefore become inefficient for both individuals as well as the drivers to reach their destination.

\subsection{Role of Governing Bodies to Control Pedestrian Crossings}

Visiting all the aspects of issues and solutions that are developed to improve the road infrastructure and pedestrian issues, the responsibility of the government comes into the scenario. In this section of the study, the government of India, as well as other organizations that tend to improve the traffic conditions, is to be involved in the project of development. Since the development of the mechanisms of crossing roads required constructions therefore, a sufficient amount of funding is to be required. These findings are either provided by the government or other assigned organizations. According to the various issues and accidents that have been witnessed due to the ineffective of pedestrian management, the governing bodies are suggested to approve for the mentioned approach ${ }^{[15]}$. Several programs are suggested to be developed by the government bodies to aware the individuals to use pedestrian crossings instead of direct crossing over the road, especially during peak hours.

\subsection{Application of Pedestrian Crossings}

According to the various observations of the occurrence of accidents, it could be suggested that the application of the mechanisms within the areas that are accident-prone, would be efficient and effective. In other words, the locations where occurs immense traffic is projected to have several alternatives for the individuals to crossroads including footbridges and subways ${ }^{[16]}$. The availability of these mechanisms would provide individuals with a secure method of crossing the roads. In addition to this, the availability of traffic personnel would also evaluate the safety measures of individuals ${ }^{[21]}$. The road infrastructure, in this case, is a very important criterion that is to be maintained effectively. In this way, the vehicles would not face congestion and thereby maintain their average speed throughout the travel. The road users, on the other hand, would be able to benefit from the effective mechanism and therefore reach their destination safely.

\subsection{Literature Gap}

The study fails to provide evidence about the affectivity of the mentioned technology for pedestrian development and controlling mechanism. There needs to be further study related to the application of the management mechanism that has been pre-stated in the study. As per the required conclusions, there has been selected a handful of research methodologies to address the issues. However, more technical aspects would be visiting in the near future research to address the problems regarding road traffic and the optimization of pedestrian crossings.

\section{METHODOLOGY}

\subsection{Research Philosophy}

Based on the current situational analysis of the traffic conditions in India, the positivist philosophy would be appropriate to apply in order to meet the objectives of the research. In other words, the reality-based statistical approach is necessary to be implemented ${ }^{[3]}$. In this way, the government of India would be able to focus on the issues that relate to the development of pedestrian crossings and controlling them to benefit road users to avail of efficient transport facilities. 


\subsection{Research Approach}

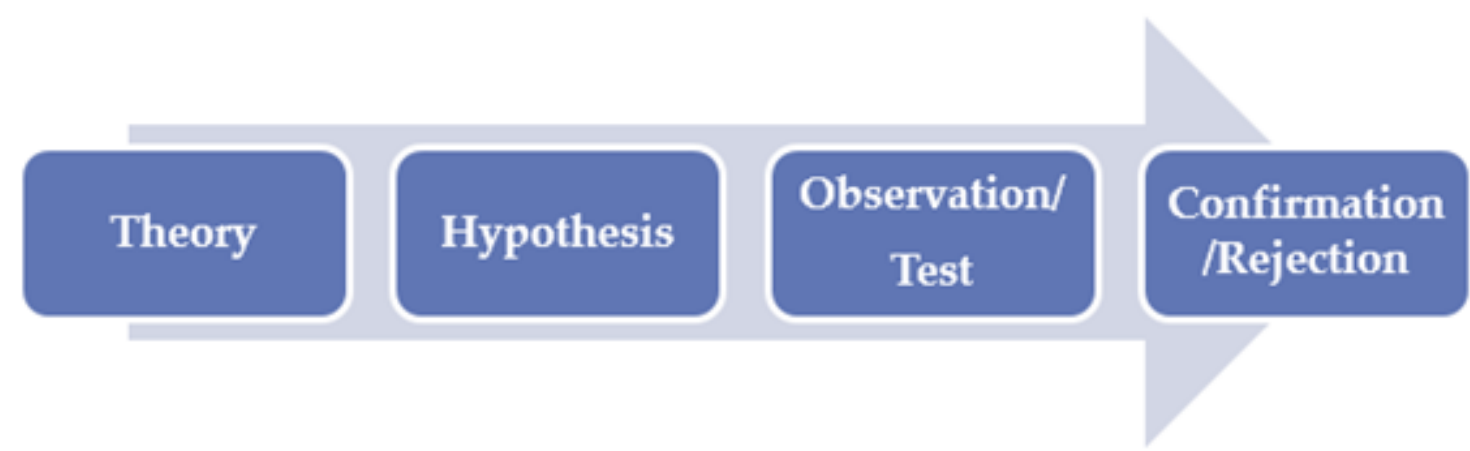

Figure 3 Deductive approach

(Source: Influenced by ${ }^{[6]}$ )

The development idea has been established are based on the situations that address pedestrian issues that are common in India. The research would be applying the deductive approach for the development strategies. Since the approach is based on pre-existing hypotheses, therefore, effective solutions would be deduced by applying the mentioned strategy ${ }^{[6]}$. The advantage of applying this research approach instead of the inductive approach is that the conclusions are derived at a very short time and therefore the applicability of the solutions to mitigate pedestrian issues would be eventually evaluated.

\subsection{Research Design}

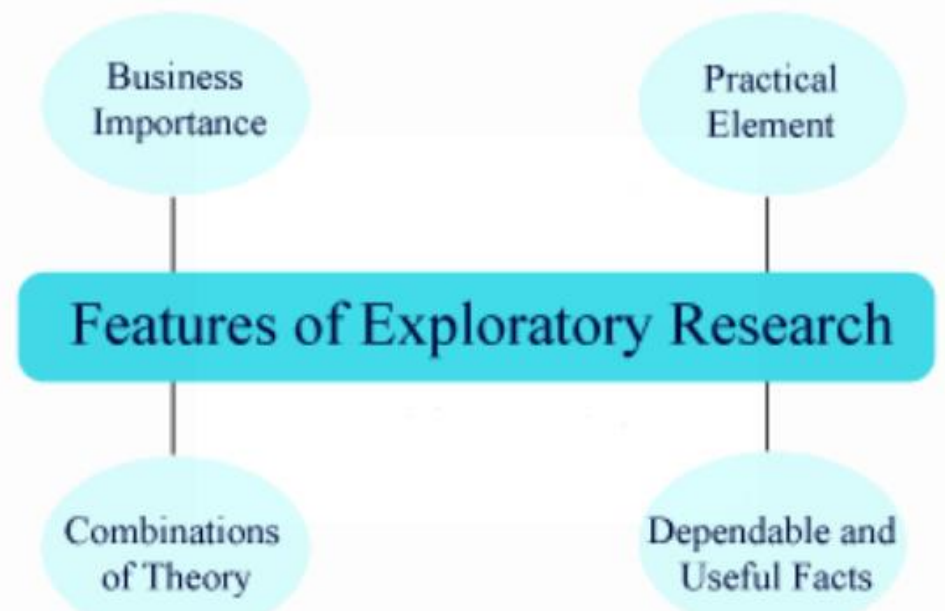

Figure 4 Exploratory Research Approach

(Source: Influenced by ${ }^{[14]}$ )

The research design that has been selected for the development procedure, in this case, is exploratory. The reason behind choosing this mechanism over other available mechanisms is that the exploratory research provides an experienced nature of the issues faced along with the alignment of the measures to solve the issues ${ }^{[14]}$. Hence, to address the issues regarding the improvement of traffic conditions through the development of pedestrian crossing conditions of India would require relevant information through surveys from various sources. These 
sources may include media or sampling of populations ${ }^{[5]}$. In this way, various perspectives of the research design would be visited and therefore would eventually be met with distinct solutions. The effective solutions along the gathered data would be combined to meet the objectives of the study as well as accomplishing the goals.

\subsection{Data Collection}

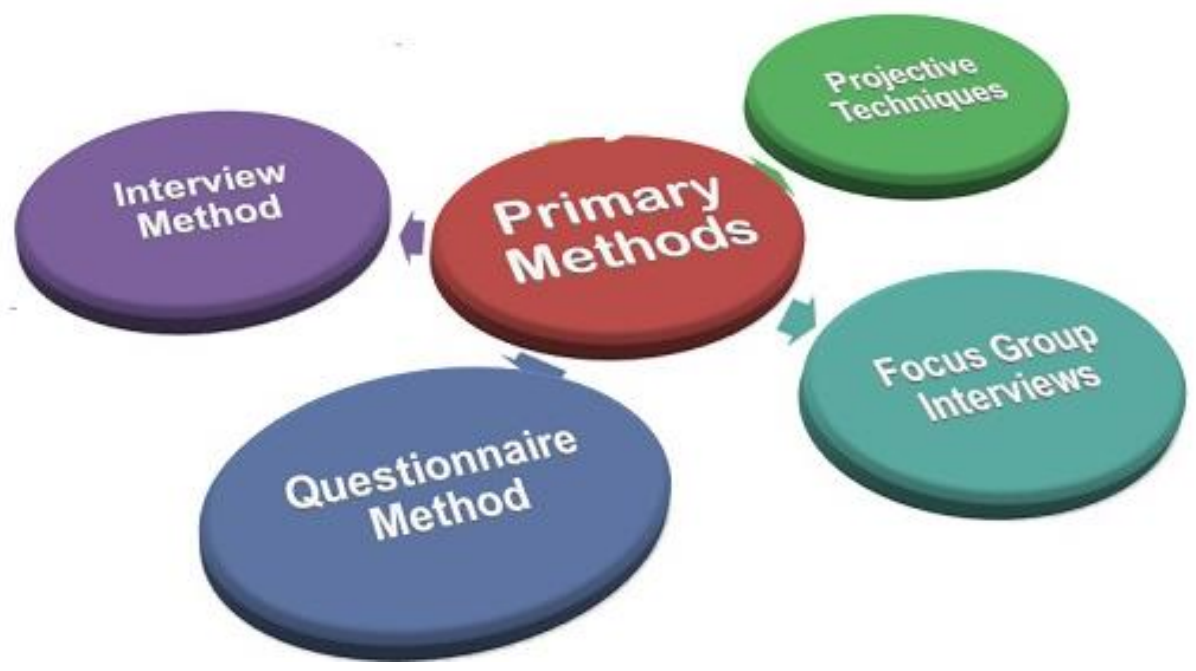

Figure 4 Primary Data collection method

(Source: Influenced by ${ }^{[23]}$ )

In the case of India, the data collection mechanism that would be applicable to be used to develop the pedestrian controlling sections would require evidence and effectively of their application accordingly. Therefore, the appropriate method would be selected as the primary data collection ${ }^{[23]}$. The advantages and benefits of applying this technique instead of secondary data is that the researchers would himself be collecting data that makes relevance to him individually. As the traffic conditions related to real-time data, therefore, the primary data collection method would facilitate the experimental approach to benefit from gaining solution measures to address the issue. In addition to this, the specific nature of the data collection method provides relevant data eliminating the chances of getting anomaly within collective measures.

\subsection{Data Processing and Analysis}

Improvement of traffic conditions in relation to the pedestrian crossing development and road infrastructure, there needs to be objective data. In other words, the data would be collected using an appropriate data collection mechanism within cloud-based database management systems ${ }^{[20]}$. Collection methods would be required to go through processing to detect the relevant information. Since the data could be gathered from various sources, there may be the presence of redundant data that is to be eliminated. The data analysis software that has been proposed to be used in big data analytics ${ }^{[2]}$. With the application of this software, the data that has been collected would be processed and all the irrelevant information within the data would be accordingly elements to sustain the release and accuracy. These data would be fetched using relevant queries ${ }^{[19]}$. These were needed to be designed by the database administrator of the proposed research. The outcomes would be eventually provided to the development bodies to initiate their processes. 


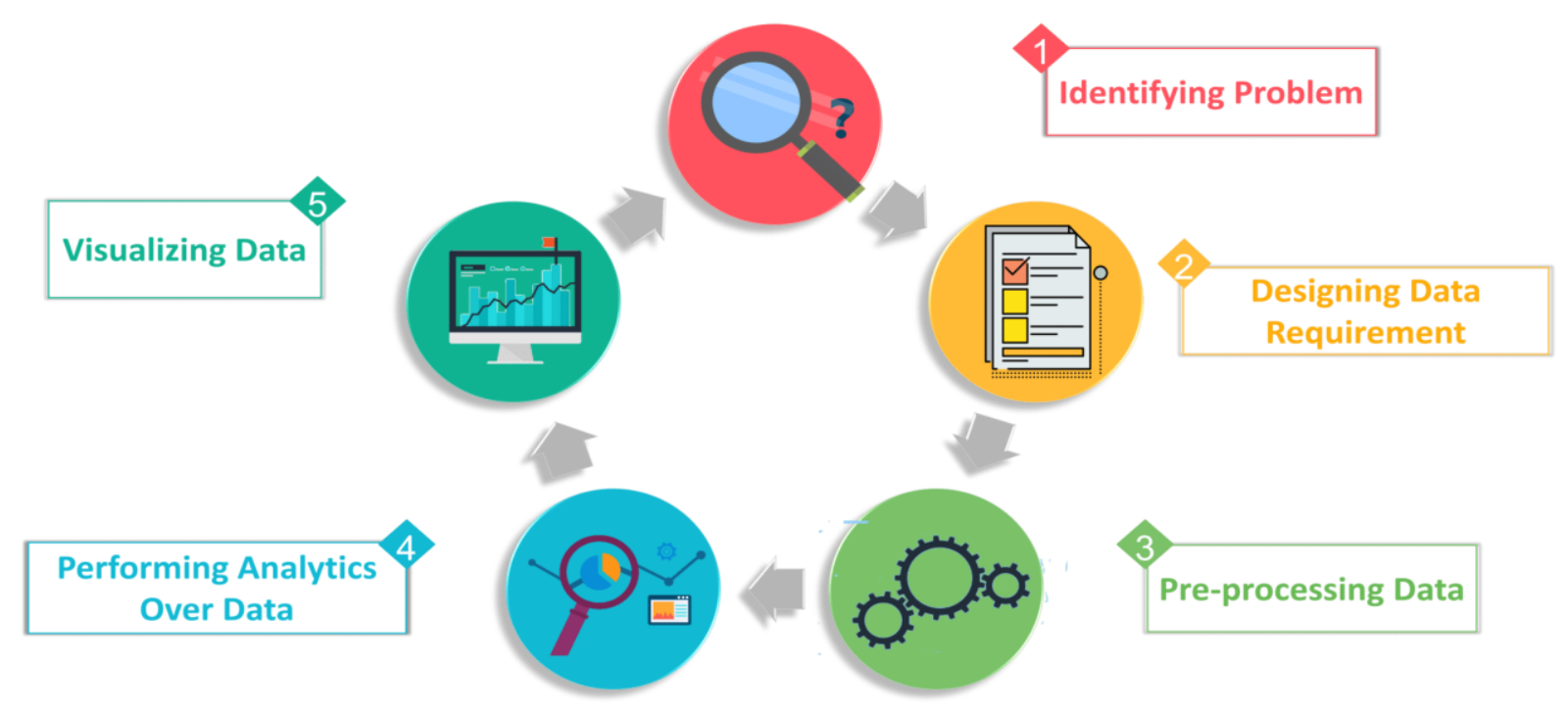

Figure 5 Data analysis

(Source: Influenced by ${ }^{[20]}$ )

\section{FINDINGS}

\subsection{Development of Footbridges}

According to the observed facts, it could be stated that places, where there exists lots of congestion, need to be facilitated with footbridge crossings. With the application of this mechanism each and every individual would be able to benefit from the safety measures of crossing roads ${ }^{[9]}$. Generally, road junctions are places where there exists a lot of traffic congestion and the police are unable to handle the situation when crowds are increased. With the development of footbridge crossing platforms, the stress over the traffic governing individuals would reduce and efficient traffic passing would be achieved. In addition to this, the individuals would benefit from time effective methods of crossing the roads.

\subsection{Acknowledgement of Individuals Regarding Traffic Rules and Regulations}

It is important to be aware of the individuals who are engaged win using roadways to travel as a daily life schedule. As observed in many cases, individuals are vulnerable to face accidents if proper measures and traffic rules are to be maintained during crossing a road ${ }^{[13]}$. With the knowledge of using the pedestrian crossings as the only medium to crossroads would eventually benefit them from maintaining the constraint of safety. Life of individuals in India has been observed to be a busy one especially near metropolitan areas such as Bangalore. Therefore, several measures by the government have been taken to safeguard the people through awareness programs stating the importance of using pedestrian crossings.

\subsection{Development of Time Determined Signal Posts}

As per the application of the time scheduled traffic management technology, it could be estimated that the technology would benefit diverse individuals. In other words, the speed of crossing a road varies from person to person which would be maintained by the device by studying the number of traffic conditions, especially during peak hours ${ }^{[8]}$. Therefore, it could be stated that modern technology, in this case, would be used by the government of India to develop this mechanism to provide the individuals and the societies with a safety measure while traveling inroads. 


\section{CONCLUSION}

Based on the various reasons regarding the development of the optimization technique of pedestrian crossings and road infrastructure, there exists a lack of safety utility for the individuals who use roadways as their daily life medium for traveling. The development of several measures to develop pedestrian crossings to safeguard the life of individuals has been established through this study. Concluding from the proposed planning of the research, upon having developed the time scheduled road crossing mechanisms, the individuals would be able to use the zebra crossing as the basic medium but with an additional feature of signal posts. In other words, people who prefer crossing roads directly using zebra crossing are said to have risky nature as there may occur accidents due to lack of efficiency in signal posts. The development of the time scheduled mechanism of signal posts would provide these individuals to improve their perceptions over road crossing and avoid being vulnerable to accidents.

\subsection{Future Scope}

In order to make roads safer for pedestrians, it is required to make the roads safer for cars. These improvements, on the other hand, would require efficient planning of road constructions and flyover developments. According to the aspects of optimization, safety measures are required to be kept in mind by the individual that is linked with the development procedures. Since India comprises a string number of population, therefore, a safety measure is to be taken as a major issue for the individuals by the government.

\subsection{Recommendations}

Based on the proposed objectives of the study, the following recommendations are provided that would be required by the developers to initiate their development procedures.

- Engage awareness among the individuals who use roadway transport as their daily life schedule

- Maintaining the traffic conditions using efficient techniques so that every vehicle remains within their speed limits and are maintained accordingly especially near the signal posts

- Development of footbridges that would reach a highly approximately above the average truck level

- Application of several modern technologies including the time scheduled traffic signal posts are to be developed in the future to maintain roadway traffic conditions accordingly

\section{REFERENCES}

[1] Angelstam P, Khaulyak O, Yamelynets T, Mozgeris G, Naumov V, Chmielewski TJ, Elbakidze M, Manton M, Prots B, Valasiuk S. Green infrastructure development at European Union's eastern border: Effects of road infrastructure and forest habitat loss. Journal of environmental management. 2017 May 15; 193:300-11.

[2] Chen L, Zheng D, Liu B, Yang J, Jin Q. VFDB 2016: hierarchical and refined dataset for big data analysis 10 years on. Nucleic acids research. 2015 Nov 17;44(D1):D694-7.

[3] Cheng Z, Tisi M. A deductive approach for fault localization in ATL model transformations. In International Conference on Fundamental Approaches to Software Engineering 2017 Apr 26 (pp. 300-317). Springer, Berlin, Heidelberg.

[4] Duim E, Lebrão ML, Antunes JL. Walking speed of older people and pedestrian crossing time. Journal of Transport \& Health. 2017 Jun 1; 5:70-6.

[5] Etikan I, Musa SA, Alkassim RS. Comparison of convenience sampling and purposive sampling. American journal of theoretical and applied statistics. 2016 Jan;5(1):1-4. 
Evaluating Various Approaches to Improve Road Infrastructure and Controlling Pedestrian

Crossings in India

[6] Grangel-González I, Collarana D, Halilaj L, Lohmann S, Lange C, Vidal ME, Auer S. Alligator: A deductive approach for the integration of industry 4.0 standards. In European Knowledge Acquisition Workshop 2016 Nov 19 (pp. 272-287). Springer, Cham.

[7] Hanan AH, Idris MY, Kaiwartya O, Prasad M, Shah RR. Real traffic-data based evaluation of vehicular traffic environment and state-of-the-art with future issues in location-centric data dissemination for VANETs. Digital Communications and Networks. 2017 Aug 1;3(3):195-210.

[8] He F, Yan X, Liu Y, Ma L. A traffic congestion assessment method for urban road networks based on speed performance index. Procedia engineering. 2016 Jan 1;137:42533.

[9] Jateikienè L, Andriejauskas T, Lingytė I, Jasiūnienè V. Impact assessment of speed calming measures on road safety. Transportation research procedia. 2016 Jan 1;14:422836.

[10] Jensen MB, Philipsen MP, Møgelmose A, Moeslund TB, Trivedi MM. Vision for looking at traffic lights: Issues, survey, and perspectives. Transactions on Intelligent Transportation Systems. 2016 Feb 3;17(7):1800-15.

[11] Kaushik PG, Dahake VD, Mahalle PV. Design of intelligent traffic light controller using fpga. International Journal of Electronics, Communication and Soft Computing Science \& Engineering (IJECSCSE). 2015;4:194.

[12] Khattak A, Tung LW. Severity of pedestrian crashes at highway-rail grade crossings. InJournal of the Transportation Research Forum 2015 (Vol. 54, No. 1424-2016-118066, pp. 91-100).

[13] Kumar S, Toshniwal D. A data mining framework to analyze road accident data. Journal of Big Data. 2015 Dec;2(1):26.

[14] Maritz A. Senior Entrepreneurship in Australia: An Exploratory Approach. International Journal of Organizational Innovation. 2015 Jan 1;7(3).

[15] Molyneaux N, Scarinci R, Bierlaire M. Pedestrian management strategies for improving flow dynamics in transportation hubs. In17th Swiss Transportation Research Conference, Ascona, Switzerland 2017 May.

[16] Oviedo-Trespalacios O, Scott-Parker B. Footbridge usage in high-traffic flow highways: The intersection of safety and security in pedestrian decision-making. Transportation research part F: traffic psychology and behaviour. 2017 Aug 1;49:177-87.

[17] Pau G, Campisi T, Canale A, Severino A, Collotta M, Tesoriere G. Smart Pedestrian Crossing Management at Traffic Light Junctions through a Fuzzy-Based Approach. Future Internet. $2018 \mathrm{Feb} ; 10(2): 15$.

[18] Pelé M, Bellut C, Debergue E, Gauvin C, Jeanneret A, Leclere T, Nicolas L, Pontier F, Zausa D, Sueur C. Cultural influence of social information use in pedestrian road-crossing behaviours. Royal Society open science. 2017 Feb 1;4(2):160739.

[19] Rödiger W, Mühlbauer T, Kemper A, Neumann T. High-speed query processing over high-speed networks. Proceedings of the VLDB Endowment. 2015 Dec 1;9(4):228-39.

[20] Tan X, Di L, Deng M, Fu J, Shao G, Gao M, Sun Z, Ye X, Sha Z, Jin B. Building an elastic parallel OGC web processing service on a cloud-based cluster: A case study of remote sensing data processing service. Sustainability. 2015 Oct;7(10):14245-58.

[21] Tarko AP. Surrogate measures of safety. InSafe Mobility: Challenges, Methodology and Solutions 2018 Apr 18 (pp. 383-405). Emerald Publishing Limited.

[22] Thurfjell H, Spong G, Olsson M, Ericsson G. Avoidance of high traffic levels results in lower risk of wild boar-vehicle accidents. Landscape and Urban Planning. 2015 Jan 1;133:98-104.

[23] Xie F, Colantonio LD, Curtis JR, Safford MM, Levitan EB, Howard G, Muntner P. Linkage of a population-based cohort with primary data collection to Medicare claims: the reasons for geographic and racial differences in stroke study. American journal of epidemiology. 2016 Sep 30;184(7):532-44. 


\section{APPENDICES}

Appendix 1: Number of Road Accident Occurrence Due to Inappropriate Management of Pedestrian Crossings all Over India

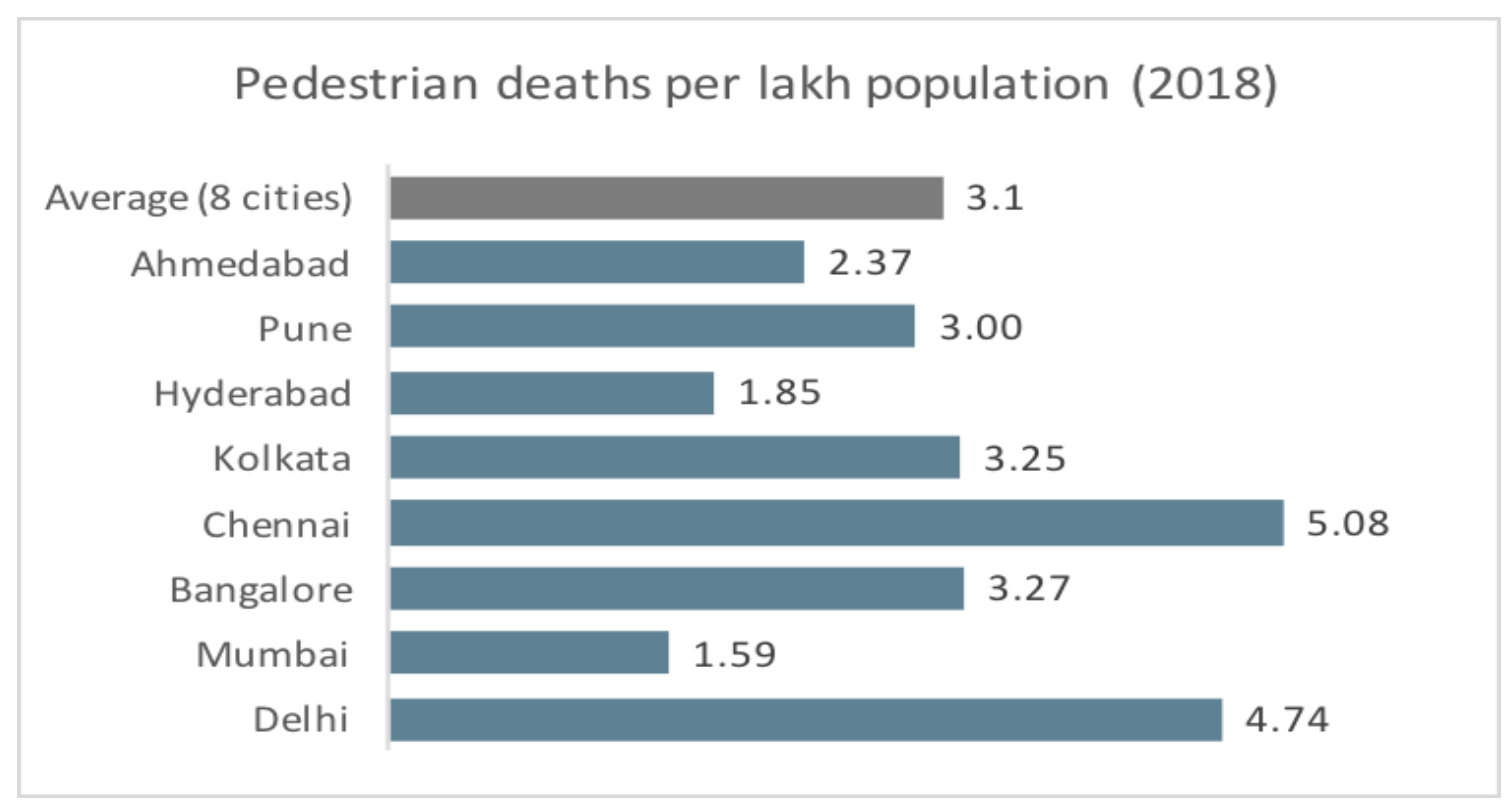

